\title{
COGNITIVE LOAD DURING ONLINE COMPLEX PROBLEM- SOLVING IN A TEACHER TRAINING CONTEXT
}

\author{
Charlotte Larmuseau, KU Leuven Campus KULAK Kortrijk, Itec-Imec, Hendrik Coucke, \\ Pamela Kerkhove, VIVES University of Applied Sciences, Piet Desmet, Fien Depaepe, KU \\ Leuven Campus KULAK Kortrijk, Itec-Imec, Belgium
}

\section{Introduction}

Online courses are becoming ubiquitous and increasingly tend to use authentic learning tasks as the driving force for teaching and learning. Nevertheless, designing online courses that incorporate real- world tasks is more challenging as these problems require more cognitive processes (van Merriënboer \& Sluijsmans, 2009). This phenomenon can be explained by Cognitive Load Theory (CLT) introduced by Sweller (1994). CLT distinguishes three types of cognitive load: intrinsic, extraneous and germane load. The level of intrinsic load is assumed to be determined by the level of element interactivity. An element can be a definition, concept, formula and procedure that needs to be or has been learned. Extraneous load is mainly imposed by instructional procedures that are suboptimal, whereas germane load refers to the learners' working memory resources available to deal with the complexity of the task or learning material (Sweller, 2010). Accordingly, the experienced cognitive load is mainly dependent of students' prior knowledge. Nevertheless, cognitive load can also be determined by students' motivation (Feldon, Franco, Chao, Peugh, \& Maahs-Fladung, 2018; Verhoeven, Schnotz, \& Paas, 2009). As a consequence, when designing an online course for complex tasks, it is important to understand how the different types of cognitive load are affected by students' cognitive and motivational characteristics. Therefore, in the current study, a high and low complex task was developed relating to the learning and teaching of geometry. The complexity of the task was manipulated by increasing the element interactivity for the high complex task (Sweller, 2010). In the low complex task one element was questioned each time, and consequently students had to apply a single rule, formula or procedure. By contrast, the high complex task was based on a real-life context (e.g., teaching geometry), and had higher element interactivity. Subsequently, the high complex task required learners to engage in a series of cognitive activities such as analysing, decision making, implementing and evaluating, while holding several procedures and rules in mind. Accordingly, we expected the high complex task to induce more cognitive load. The same amount of support containing the same content, was provided during both tasks. Consequently, in this context, students could take initiative in diagnosing their learning needs by identifying appropriate support. Since students could consult different amounts of support, this self-directed learning strategy could also influence the perceived cognitive load (van Merriënboer \& Sluijsmans, 2009). Accordingly, the amount of consulted support was also taken into account during the 


\section{Cognitive Load During Online Complex Problem-Solving in a Teacher Training Context Charlotte Larmuseau et al.}

analyses. The aim of the study was twofold. First, as a manipulation check of task complexity, we investigated differences in the experienced cognitive load while solving a high and low complex task. Secondly, we examined whether students' cognitive and motivational characteristics influence the different types of perceived cognitive load, when taking into account the amount of consulted support for both the high and low complex task. A multivariate approach was chosen to assess the degree of interplay that may exist among students' cognitive, motivational characteristics, consultation of support and the different types of perceived cognitive load. By conducting this study, we wanted to gain insight into whether the cognitive, motivational characteristics and consultation of support influence the perceived cognitive load differently for a high and low complex task.

\section{Theoretical framework}

CLT is a broadly applied theory within the field of instructional design for complex learning (Sweller, 2010). CLT uses current knowledge about the human cognitive architecture as a baseline. Basically, the human cognitive architecture consists of an effectively unlimited longterm memory, which interacts with a working memory that has limited processing capacity (Sweller, 1994). Long-term memory contains cognitive schemata that are used to store and organize knowledge. Learning occurs when information is successfully processed in working memory and when new schemas are created or incorporated into consisting schemas in longterm memory. As the processing capacity of the working memory is limited, overcoming individual working memory limitations by instructional manipulations has been the main focus of CLT (Sweller, 2010). According to CLT, cognitive load can be defined as a multidimensional construct representing the load that solving a particular problem imposes on the learners' cognitive system (Leppink, Paas, van der Vleuten, van Gog, \& van Merriënboer, 2013). Specifically, CLT claims that the cognitive load that learners experience can be intrinsic, extraneous or germane as indicated in Figure 1. For effective learning to occur, the sum of these loads must remain smaller than the capacity of the learner's working memory (Sweller, 1994). Intrinsic load involves element activity which is determined by the nature of the task demands in relation to the expertise and motivation of the learner. Instructional design may result in extraneous load (which is ineffective for learning) and in germane load (which is effective for learning). Extraneous cognitive load is defined as unnecessary extra load due to poorly designed instruction. Germane load is defined as load that contributes to learning (Sweller, 2010).

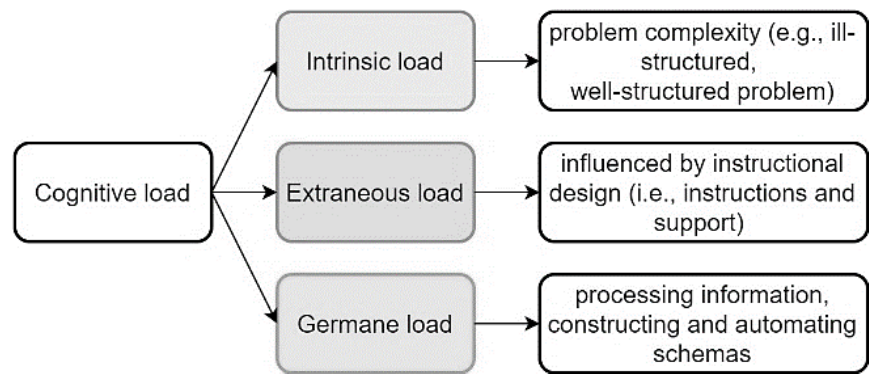

Figure 1. Overview theoretical framework 
From the perspective of CLT, the major factor influencing an individual's success in learning from instruction is the limited ability of working memory to assimilate and structure target information (Sweller, 2010). The availability of relevant well-structured prior knowledge can increase the functional capacity of working memory relative to the task, such that an individual with greater expertise will experience a lower burden on working memory resources than an individual with less expertise. Research within the CLT framework also engages motivation as a necessary precursor to learning (Paas, Tuovinen, van Merriënboer, \& Darabi, 2005; Verhoeven et al., 2009). CLT research studies assume that sufficient motivation is required for participants to invest the mental effort necessary to meet the cognitive demands of instruction. Self-efficacious learners believe that they can accomplish a task. There is evidence that self-efficacious learners tend to demonstrate a more strategic approach to learning tasks and direct mental effort toward processes that are more pertinent to learning (Feldon et al., 2018). Task value essentially refers to the reason for doing a task. More specifically, students with high task value pursue enjoyment of learning and understanding of new things. The level of motivation can be particularly important if high complex tasks need to be solved as a higher degree of cognitive demand imposes higher cognitive load and requires greater effort (Paas et al., 2005). CLT furthermore indicates that the cognitive demands within a learning situation are defined by the complexity of the problem (Verhoeven et al., 2009). Nevertheless, in interactive online learning environments, students can often consult additional support that may moderate the level of complexity (van Merriënboer \& Sluijsmans, 2009). Van Merriënboer (1997) designed a four-component instructional design model (4C/ID-model) that has proven to be useful to design complex problem-solving instruction. Van Merriënboer (2013) claims that in this model cognitive load can be managed by providing a large amount of support and guidance. Accordingly, learners are more guided into how to solve the complex problem and accordingly spend less time on irrelevant aspects of the task. In the 4C/ID-model two types of support are incorporated, namely procedural and supportive information. Procedural information specifies how to solve the routine aspects of the problem. Supportive information is basically, the theory and supports the learning and performance of the non-routine problem solving and reasoning aspects. Even though this model is more focused on the instructional design of educational programmes than the design of instructional materials, it offers directions in reducing extraneous as well as intrinsic cognitive load (van Merriënboer \& Sluijsmans, 2009). Against this theoretical background, following research questions were formulated.

- RQ1: Does the manipulation of the level of complexity of a task based on element interactivity, result in differences in perceived cognitive load between a high and low complex task?

- RQ2: Does students' cognitive and motivational characteristics influence the perceived cognitive load of a high and low complex task, when taking into account the amount of consultation of support? 


\section{Methodology}

Participants were 70 future primary school teachers of which 56 were female and 14 male (age between 18-24). All participants were first year bachelor students (i.e., second semester). The study was highly ecologically valid as the study was orchestrated by the students' lecturer of the teaching mathematics course unit. Moreover, the intervention was integrated into the students' study program (i.e., primary school teacher training). The intervention consisted of a within-subject design and was conducted online in the Moodle learning management system (LMS). The intervention took place in the auditorium of the students' faculty where students could solve the tasks individually on their own computer among their fellow students. This session was supervised by their lecturer and a researcher. Students first received an online questionnaire where students' self-efficacy was measured. Next, all students had to solve a high complex and a low complex task on preparing a lesson in geometry. After each condition, cognitive load was measured. In order to control for order effects, (a) half of the subjects were exposed to the high complex task during the first session and the low complex task during the second session, whereas for (b) the other half, the sequence was vice versa.

\section{High and low complex problem}

The high and low complex task were developed in Moodle. The content of both tasks was preparing a lesson on the circumference of a circle. Students had not yet been taught about teaching the circumference of the circle. Both tasks contained six elements where aspects of pedagogical content knowledge (PCK, i.e., inductive teaching strategy, selecting adequate lesson material and using it in a correct manner, aligning the topic of the lesson with the Flemish curriculum and taking into account differentiation in the classroom) and content knowledge (CK; i.e., formula of the circumference of the circle) were addressed. The difference between the high and low complex task was that in the high complex task students had to coordinate and integrate six elements consisting of CK and PCK in order to write a course preparation about the circumference of the circle, whereas the low complex task consisted of six questions where each element was addressed separately. During both problems, the same support consisting of procedural and supportive information was provided. Supportive information is much more extensive (i.e., background theory). Both procedural and supportive information could be consulted by clicking on the words in italics, during the complex problem-solving process. Students spent on average $25.82 \mathrm{~min}$ $(S D=6.82)$ on the high complex task and $8.04 \mathrm{~min}(S D=2.57)$ on the low complex task. Students were assessed concerning the aforementioned six items (e.g., inductive teaching). Task performance was $56.7 \%(S D=31.2)$ for the high complex task and $75.7 \%(S D=22.6)$ for the low complex task.

\section{Measurements}

For the measurement of cognitive load, a validated instrument for intrinsic, extraneous and germane load in complex knowledge domains was used as originally developed by Leppink et al. (2013). Self-efficacy (5 items) and task-value (3 items) were retrieved from the motivated 
strategies for learning questionnaire (MSLQ; Duncan \& Mckeachie, 2005). Questionnaires consisted of a 7-point Likert scale (i.e., ranging from totally disagree to totally agree). Construct validity was checked by conducting a confirmatory factor analysis (CFA). The standardized factor loadings from the latent variable constructs were all significant with standardized values ranging from .42 to .93. Internal consistency was investigated by measuring Cronbach's Alpha [Self-efficacy: .88; Task Value: 67; Low complex: intrinsic load: .86; extraneous load: .72 and germane load: .79; High complex: intrinsic load: 75, extraneous load: .73 and germane load: .80], indicating medium/good reliability (Schreiber, Nora, Stage, Barlow, \& King, 2006). Sample items are:

- intrinsic load: "the topics covered in this problem were very complex";

- extraneous load: "the instructions were very unclear";

- germane load: "The task really enhanced my understanding of the topics covered";

- $\quad$ self-efficacy: "I'm certain I can understand the most difficult material presented in this online course";

- task value: "I am very interested in the content of this course".

Information about students' prior knowledge was gathered in the first semester during their examination. Students were tested on their knowledge of PCK (mean $=57.4 \%, S D=18$ ) and $\mathrm{CK}$ (mean $=45 \%, S D=24)$. Content was (teaching) mathematics in general and geometry in particular. The amount of consulted support was collected by tracking students' activity, namely, the registration of views by the Moodle LMS. Specifically, these are the set of variables that were included: (a) amount of consultation of support during the high complex task (mean $=6.33, S D=1.75, \min =0, \max =27$ ) and $(\mathrm{b})$ the amount of support during the low complex task (mean $=3.84, S D=2.92, \min =0, \max =13$ ).

\section{Results}

$R Q 1$ investigates whether the manipulation of the level of complexity of a problem results in differences in perceived cognitive load. Results reveal that the perceived intrinsic load and extraneous load is significantly higher for the high complex problem. This indicates that students perceived the high complex task as more difficult. Germane load is significantly lower for the high complex task indicating that it was harder for students to learn from the high complex task.

Table 1: $\quad$ Paired Samples Test comparing differences of cognitive load between a high and low complex task

\begin{tabular}{lcccc}
\hline & Mean difference & $S D$ & $t$ & $p$ \\
\hline Intrinsic load & .59 & 1.08 & 4.48 & .00 \\
Extraneous load & .54 & .97 & 4.65 & .00 \\
Germane load & -.29 & 1.19 & -2.04 & .05 \\
\hline
\end{tabular}

RQ2 investigates how students' cognitive and motivational characteristics influence the different types of perceived cognitive load in a high and low complex task. Results of a multivariate regression for the high complex task reveal that less self-efficacious students perceive more intrinsic load. Partial $\eta 2$, which is the proportion of variance accounted for by 


\section{Cognitive Load During Online Complex Problem-Solving in a Teacher Training Context \\ Charlotte Larmuseau et al.}

an effect, indicates that $29 \%$ of all variance in perceived intrinsic load is attributable to students' level of self-efficacy. Results furthermore indicate a positive significant influence of the amount of support consulted on perceived intrinsic load. Partial $\eta 2$ indicates that $8 \%$ of all variances in perceived intrinsic load is attributable to the consultation of support. Results reveal that students' characteristics have no significant influence on perceived extraneous and germane load. Finally, results reveal that consulting support has a positive influence on germane load. Partial $\eta 2$ indicates that $11 \%$ of all variances in perceived germane load is the result of the consultation of support. In the low complex condition, results reveal that students' self-efficacy has a significant influence the perceived intrinsic load. Partial $\eta 2$ indicates that $11 \%$ of all variances in perceived intrinsic load is due to students' self-efficacy. Students' characteristics had no influence on the perceived extraneous and germane load. Nevertheless, results indicate a positive significant influence of the consultation of procedural information on perceived extraneous load.

Table 2: Influence of students' characteristics on the perceived cognitive load in a high complex condition

\begin{tabular}{llcccc}
\hline High complex & & $B$ & $S E$ & $p$ & $\eta_{p}{ }^{2}$ \\
\hline Intrinsic load & Intercept & 4.76 & .73 & .00 & .44 \\
& Prior knowledge & .09 & .07 & .18 & .03 \\
& Self-efficacy & -.75 & .16 & .00 & .29 \\
& Task value & .22 & .17 & .21 & .03 \\
& Amount of support & .06 & .03 & .03 & .08 \\
Extraneous load & Intercept & 4.67 & .82 & .00 & .38 \\
& Prior knowledge & -.09 & .07 & .22 & .03 \\
& Self-efficacy & -.27 & .18 & .15 & .00 \\
& Task value & -.04 & .19 & .85 & .04 \\
& Amount of support & .04 & .03 & .16 & .01 \\
Germane load & 1.13 & 1.06 & .29 & .02 \\
& Intercept & -.06 & -.60 & .55 & .01 \\
& Prior knowledge & .30 & 1.28 & .21 & .03 \\
& Self-efficacy & .31 & 1.26 & .21 & .03 \\
& Task value & .10 & .04 & .01 & .11 \\
\hline & Amount of support & .10
\end{tabular}

Table 3: Influence of students' characteristics on the perceived cognitive load in a low complex task condition

\begin{tabular}{llcccc}
\hline low complex & & $B$ & $S E$ & $p$ & $\eta_{p}{ }^{2}$ \\
\hline Intrinsic load & Intercept & 4.06 & .91 & .00 & .27 \\
& Prior knowledge & -.02 & .08 & .86 & .00 \\
& Self-efficacy & -.61 & .21 & .01 & .14 \\
& Task value & .32 & .22 & .15 & .04 \\
& Amount of support & .03 & .05 & .52 & .01 \\
Extraneous load & Intercept & 3.47 & .78 & .00 & .27 \\
& Prior knowledge & -.07 & .07 & .34 & .02 \\
& Self-efficacy & -.20 & .18 & .26 & .00 \\
& Task value & .02 & .19 & .92 & .00 \\
& Amount of support & .10 & .04 & .02 & .09 \\
Germane load & Intercept & 3.72 & .90 & .00 & .24 \\
& Prior knowledge & -.02 & .08 & .84 & .00 \\
& Self-efficacy & -.03 & .21 & .91 & .00 \\
& Task value & .07 & .22 & .76 & .00 \\
& Amount of support & .04 & .05 & .40 & .01 \\
\hline & & & & &
\end{tabular}




\section{Discussion}

$R Q 1$ investigated the manipulation of complexity of the conditions based on element interactivity. Results reveal that the students indicate higher perceived intrinsic load for the high complex task when compared with the low complex task. This indicates that the manipulation of complexity based on element interactivity was successful. Additionally, results indicate that extraneous cognitive load was significantly higher for the high complex task. Nevertheless, the instructions for both conditions were of the same level of difficulty. The difference in extraneous load could be the result of differences in students' problemsolving strategies across the high and low complex task. Former studies indicated that problem-solving strategies such as selecting and processing relevant information, can induce extraneous cognitive load (Boekaerts, 2017; Likourezos \& Kalyugo, 2017). Finally, results reveal a significant higher germane load for the low complex task, indicating that students found it easier to learn from the low complex task. Findings indicate that a task with higher element interactivity requires more cognitive processes. As a result, there are not always enough cognitive resources available to learn (e.g., process information) from such complex tasks (Sweller, 2010). RQ2 examined the influence of students' cognitive, motivational and consultation of support on the different types of cognitive load for a high and low complex task. Results of the high complex condition reveal that more self-efficacious students experience less intrinsic cognitive load. These results indicate that students who believe in their capability to solve the complex problem perceive the task as less complex. Moreover, students' self-efficacy seems to exert more influence on perceived intrinsic load when compared with students' prior knowledge and students' task value. These preliminary results therefore emphasize the importance of students' self-efficacy when investigating cognitive load (Paas et al., 2005). Results furthermore indicate that students who consulted more support, perceived higher intrinsic load. From a CLT perspective, we would rather assume that consulting support reduces cognitive load. More specifically, we would assume that students might have tried to compensate for their lack of prior knowledge by consulting support (Larmuseau, Elen, \& Depaepe, 2018). In this study, it is not entirely clear whether students have consulted support to compensate for the complexity of the task, or whether the consultation (and maybe the complexity) of the support has increased their cognitive load. Findings reveal that consulting support also had a positive influence on germane cognitive load. This actually means that the support helped the students to learn from the high complex task. When we combine all findings, we can deduce that students consulted the support when they probably lacked the knowledge to solve the high complex task and that, thanks to this support, they were able to learn new things. When investigating the low complex task, results also reveal that more self-efficacious students perceive less intrinsic cognitive load, nevertheless the effect size is smaller when compared with the high complex task, indicating that students' self-efficacy is even more important when task complexity increases (i.e., higher element interactivity). Findings also reveal that consulting support induced perceived extraneous load. Again, the direction of the effect of the consultation of support is not completely clear. Intuitively, we would think that the lack of clarity of instructions caused 
students to seek more support. On the other hand, we do not immediately see a link between the consulted support and the perceived intrinsic load, which might indicate that the low complex task was easier (i.e., which also became clear from the findings or RQ1) and that the extra support induced extraneous load, as this might have been redundant (Boekaerts, 2017).

\section{Conclusions and further research}

Results reveal that students' self- efficacy has a major influence on the perceived intrinsic load for both the high and low complex task. Additionally, results of the current study indicate that students' level of self-efficacy is even more important than students' prior knowledge and task value for both the high and low complex task. The consultation of support also influences perceived intrinsic and germane load for the high complex task, and perceived extraneous load for the low complex task. Due to the study design it is not completely clear whether students have consulted support to mediate their experienced cognitive load, or whether the consultation of support induced intrinsic or extraneous cognitive load. To gain insight into that process for both the high and low complex task, it is interesting to perform repeated measurements of self-reported cognitive load in follow-up studies during online complex problem solving. In addition, it would be an added value to incorporate continuous measures of cognitive load and relate differences to actual problem-solving behaviour (Larmuseau, Desmet, Vanneste, \& Depaepe, 2019).

\section{References}

Boekaerts, M. (2017). Cognitive load and self-regulation: Attempts to build a bridge. Learning and Instruction, 51, 90-97. https://doi.org/10.1016/j.learninstruc.2017.07.001

Duncan, T. G., \& McKeachie, W. J. (2005). The making of the motivated strategies for learning questionnaire. Educational Psychologist, 40, 117-128 https://doi.org/10.1207/s15326985ep4002_6

Feldon, D. F., Franco, J., Chao, J., Peugh, J., \& Maahs-Fladung, C. (2018). Self-efficacy change associated with a cognitive load-based intervention in an undergraduate biology course. Learning and Instruction, 56, 64-72. https://doi.org/10.1016/j.learninstruc.2018.04.007

Larmuseau, C., Elen, J., \& Depaepe, F. (2018). The influence of students' cognitive and motivational characteristics on students' use of a 4C/ID-based online learning environment and their learning gain. Proceedings of the $8^{\text {th }}$ International Conference on Learning Analytics and Knowledge - LAK '18. https://doi.org/10.1145/3170358.3170363

Larmuseau, C., Desmet, P., Vanneste, P. \& Depaepe, F. (2019). Multichannel data for understanding cognitive affordances during complex problem solving. Proceedings of the $8^{\text {th }}$ International Conference on Learning Analytics and Knowledge - LAK '19. https://doi.org/10.1145/3303772.3303778

Leppink, J., Paas, F., van der Vleuten, C. P. M., van Gog, T., \& van Merriënboer, J. J. G. (2013). Development of an instrument for measuring different types of cognitive load. Behavior Research Methods, 45, 1085-1072. https://doi.org/10.3758/s13428-013-0334-1 
Likourezos, V., \& Kalyuga, S. (2017). Instruction-first and problem-solving-first approaches: alternative pathways to learning complex tasks. Instructional Science, 45, 195-219. https://doi.org/10.1007/s11251-016-9399-4

van Merriënboer, J. J. G. (1997). Training complex cognitive skills: a four-component instructional design model for technical training. Englewood Cliffs, NJ: Educational Technology Publications.

van Merriënboer, J. J. G. (2013). Perspectives on problem solving and instruction. Computers and Education, 64, 153-160. https://doi.org/10.1016/j.compedu.2012.11.025

van Merriënboer, J. J. G., \& Sluijsmans, D. M. A. (2009). Toward a synthesis of cognitive load theory, four-component instructional design, and self-directed learning. Educational Psychology Review, 21, 55-66. https://doi.org/10.1007/s10648-008-9092-5

Paas, F., Tuovinen, J. E., van Merriënboer, J. J. G., \& Darabi, A. A. (2005). A motivational perspective on the relation between mental effort and performance: Optimizing learner involvement in instruction. Educational Technology Research and Development, 53, 25-34. https://doi.org/10.1007/BF02504795

Schreiber, J. B., Nora, A., Stage, F. K., Barlow, E. A., \& King, J. (2006). Reporting structural equation modeling and confirmatory factor analysis results: a review. The Journal of Educational Research, 99, 323-338. https://doi.org/10.3200/JOER.99.6.323-338

Sweller, J. (1994). Cognitive load theory, learning difficulty, and instructional design. Learning and Instruction, 4, 295-312. https://doi.org/10.1016/0959-4752(94)90003-5

Sweller, J. (2010). Element interactivity and intrinsic, extraneous, and germane cognitive load. Educational Psychology Review, 22, 123-138. https://doi.org/10.1007/s10648-010-9128-5

Verhoeven, L., Schnotz, W., \& Paas, F. (2009). Cognitive load in interactive knowledge construction. Learning and Instruction, 19, 369-375.

https://doi.org/10.1016/j.learninstruc.2009.02.002 